\title{
Risk factors for Mycobacterium ulcerans infection (Buruli Ulcer) in Togo - a case- control study in Zio and Yoto districts of the maritime region
}

\author{
Issaka Maman ${ }^{1,2^{*}}$ D, Tchadjobo Tchacondo ${ }^{2}$, Abiba Banla Kere ${ }^{1}$, Ebekalisai Piten ${ }^{3}$, Marcus Beissner ${ }^{4}$, \\ Yiragnima Kobara ${ }^{5}$, Komlan Kossi ${ }^{1}$, Kossi Badziklou ${ }^{1}$, Franz Xaver Wiedemann ${ }^{6}$, Komi Amekuse ${ }^{6}$, Gisela Bretzel ${ }^{4}$ \\ and Damintoti Simplice Karou ${ }^{2}$
}

\begin{abstract}
Background: Buruli ulcer (BU) is a neglected mycobacterial skin infection caused by Mycobacterium ulcerans. This disease mostly affects poor rural populations, especially in areas with low hygiene standards and sanitation coverage. The objective of this study was to identify these risk factors in the districts of Zio and Yoto of the Maritime Region in Togo.

Methods: We conducted a case-control study in Zio and Yoto, two districts proved BU endemic from November 2014 to May 2015. BU cases were diagnosed according to the WHO clinical case definition at the Centre Hospitalier Régional de Tsévié (CHR Tsévié) and confirmed by Ziehl-Neelsen (ZN) microscopy and IS2404 polymerase chain reaction (PCR). For each case, up to two controls matched by sex and place of residence were recruited. Socio-demographic, environmental or behavioral data were collected and conditional logistic regression analysis was used to identify and compare risk factors between BU cases and controls.
\end{abstract}

Results: A total of 83 cases and 128 controls were enrolled. The median age was 15 years (range 3-65 years). Multivariate conditional logistic regression analysis after adjustment for potential confounders identified age $(<10$ years $(\mathrm{OR}=11.48$, $95 \% \mathrm{Cl}=3.72-35.43)$ and $10-14$ years $(\mathrm{OR}=3.63,95 \% \mathrm{Cl}=1.22-10.83))$, receiving insect bites near a river $(\mathrm{OR}=7.8,95 \%$ $\mathrm{Cl}=1.48-41.21)$ and bathing with water from open borehole $(\mathrm{OR}=5.77,(1.11-29.27))$ as independent predictors of acquiring $\mathrm{BU}$ infection.

Conclusions: This study identified age, bathing with water from open borehole and receiving insect bites near a river as potential risk of acquiring BU infection in Zio and Yoto districts of the Maritime Region in south Togo.

Keywords: Buruli ulcer, Mycobacterium ulcerans, District of Zio, District of Yoto, Togo, Risk factor, Case-control study

\section{Background}

Buruli ulcer (BU) is an emerging skin disease caused by an infection with Mycobacterium ulcerans [1-4]. BU represents the third most common mycobacterial disease after tuberculosis and leprosy in immunocompetent hosts. Infection with M. ulcerans often leads to

\footnotetext{
* Correspondence: mamanissaka@yahoo.fr

'Institut National d'Hygiène (INH), National Reference Laboratory for Buruli ulcer disease in Togo, 26 QAD Rue Nangbeto, 1BP, 1396 Lomé, Togo

${ }^{2}$ Ecole Supérieure des Techniques Biologiques et Alimentaires (ESTBA),

Laboratoire des Sciences Biologiques et des Substances Bioactives, Université de Lomé, Lomé, Togo

Full list of author information is available at the end of the article
}

extensive destruction of skin and soft tissue with the formation of large ulcers, commonly on limbs. About $60 \%$ of lesions occur on the lower limbs, $30 \%$ on the upper limbs and $10 \%$ on the rest of the body. Although the rate of mortality of Buruli ulcer is low, the serious morbidity caused by the disease includes functional disabilities that may result in permanent social, economic and developmental problems. At least $50 \%$ of those affected by BU are children aged $<15$ years. Rate of infections among males and females are equal [1-5]. To date, BU cases have been reported in over 30 countries, particularly in tropical and subtropical climate regions but also in 
temperate climate zones such as Japan and southern Australia [1-5]. BU is a neglected tropical disease (NTD) with a poorly known global prevalence and mainly affects remote rural African communities [6]. According to the $\mathrm{WHO}$, from an estimated $7000 \mathrm{BU}$ cases reported annually (2016) worldwide and more than 4000 cases occurred in Sub-Saharan Africa. The largest numbers of reported BU cases were from West African countries, particularly from Ivory Coast (about 2000 cases annually), Benin and Ghana as well, each of which reported about 1000 cases a year (2016) [1-6].

In Togo, the first cases of BU have been described in 1996 by Portaels et al. [7]. From 1996 to 2004, more than 100 cases were clinically diagnosed $[8,9]$. Between 2007 through 2010 [9], a joint research project between the German Leprosy and Tuberculosis Relief Organization in Togo (DAHWT) and the Department for Infectious Diseases and Tropical Medicine, University Hospital, Ludwig Maximilians-University, Munich, (Germany) allowed the first systematic study of laboratory confirmed BU cases from Togo and established prevalence of BU in the Maritime Region of south Togo. Since 2011, within the frame of the European Community funded research project "BuruliVac", a National Reference Laboratory for BU (NRL-UB) was established at the Institut National d'Hygiène (INH) and all BU cases notified were confirmed by IS2404 PCR [10].

Previous case-control studies [11-15] have reported a high risk of contracting Buruli ulcer by swimming in or wading through a river. Residence near marshy areas with stagnant or slow-flowing water bodies and farming activities near rivers were additionally described as risk factors [11-15]. Several epidemiologic studies in Africa [16-19] and Australia [20, 21] have identified aquatic sources as possible reservoirs of $M$. ulcerans by detecting DNA of the pathogen in water filtrant and in a range of environmental samples. All these findings used PCR methodology which does not provide definitive proof for the presence of intact bacteria in a matrix. More recently, results from laboratory experiments [22-25] have suggested a new hypothesis that aquatic insects, fish, plants and terrestrial mammals may be reservoirs for $M$. ulcerans and that insect may be even involved in transmission to humans. In addition, the successful culture of M. ulcerans from an aquatic water bug collected in Benin [26] provides definitive evidence for the presence of $M$. ulcerans in an aquatic invertebrate as possible reservoirs or vectors of $M$. ulcerans. This considerable achievement showed that the M. ulcerans is present in the environment and that transmission to humans might occur through contact with water or environmental samples contaminated with or harboring the mycobacteria [27]. Inoculation of this pathogen into the subcutaneous tissue could occur when the exposed skin is traumatized.
However, the exact mechanism of transmission of the bacterium remains unclear [27].

Human-linked changes in the aquatic environment such as dam constructions on rivers, deforestation, agriculture and mining have led to environmental disturbance and may contribute to the spread of $M$. ulcerans $[28,29]$. This could increase the incidence of Buruli ulcer cases in endemic areas and lead to the emergence of $M$. ulcerans in areas where the pathogen was previously absent [28]. Some studies, mainly clinical [7-10, 30-32], were carried out in Togo on BU but little were focused on socio-demographic, environmental or behavioral factors. We conducted this study to determine such risk factors for M. ulcerans infection in the Zio and Yoto Districts in the Maritime Region.

\section{Methods \\ Study design}

We conducted a case-control study in the Zio and Yoto districts of the maritime region (Fig. 1) between November 2014 and May 2015. Buruli ulcer cases were selected at the National Reference Center for BU Treatment (CNRT-UB) located at CHR Tsévié. Patients enrolled were recruited from March 2013 to May 2015. Controls were recruited by active search during the survey. Patients infected with the human immunodeficiency virus (HIV) or with active tuberculosis were excluded from the study.

\section{Case definition}

A probable case of Buruli ulcer was defined as any person aged $\geq 2$ years who lived in Zio or Yoto district showing clinical symptoms according to the WHO clinical definition of BU [3]. A confirmed case was defined as a probable case with detection of $M$. ulcerans using Ziehl-Neelsen (ZN) microscopy and IS2404 PCR $[9,10]$.

\section{Recruitment of controls}

An eligible control was defined as any person aged $\geq 2$ years without any history or clinical symptoms of Buruli ulcer. Up to two controls were randomly selected and matched to cases by sex and place of residence (home where lived the case or neighbor home in the same village).

\section{Study site}

This study was conducted in 17 villages in districts of Zio and Yoto where more than $85 \%$ of confirmed BU patients originate. These districts are in the Maritime Region (South of Togo) which covers an area of $6.359 \mathrm{~km}^{2}$. With an estimated population of $1,762,518$ inhabitants in 2012, the climate is tropical and humid with two rainy seasons and two dry seasons. The maritime region has a flat topography, with a low contrast characterized by a sedimentary basin that covers $4 / 5$ of 


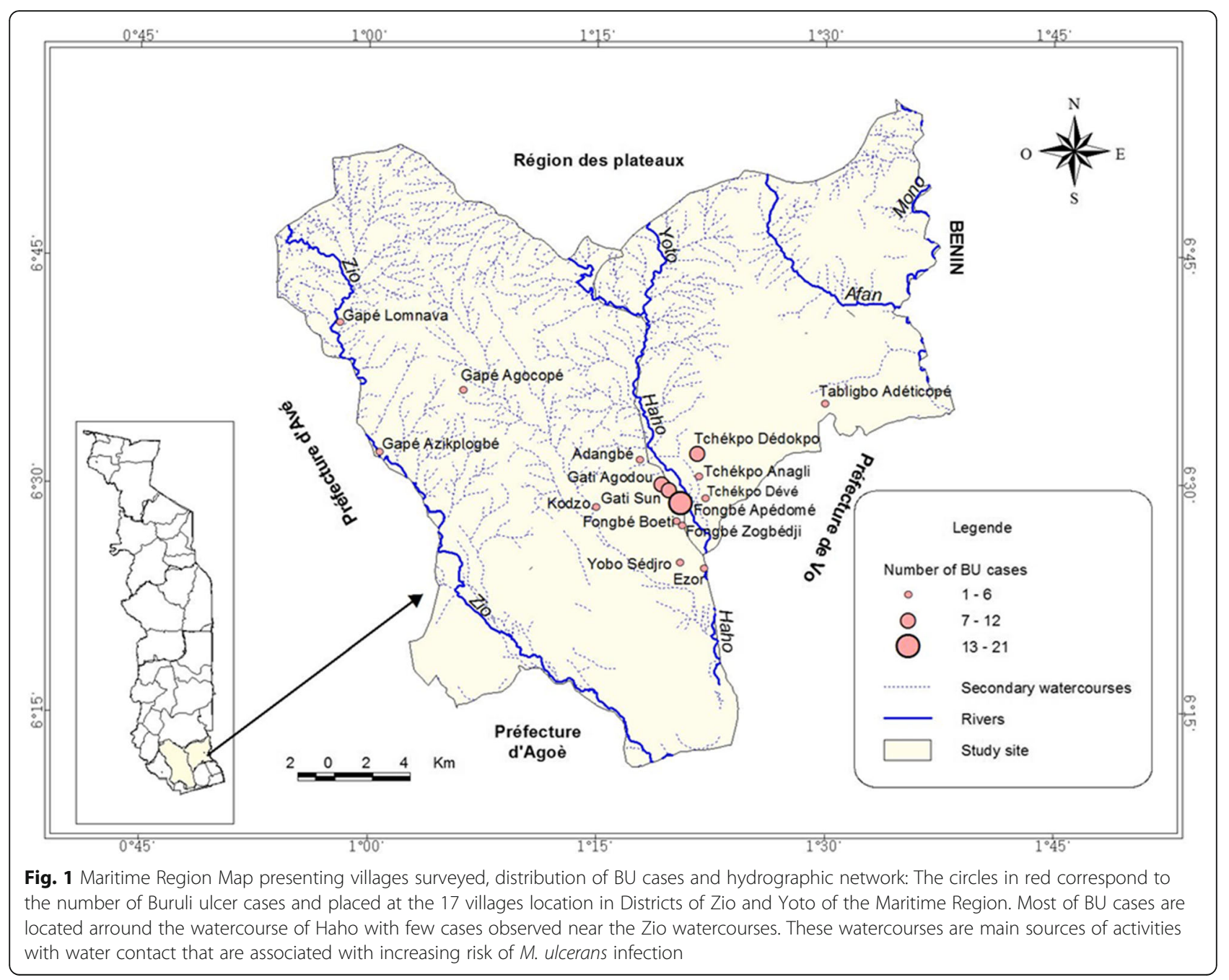

the region, a low altitude (50-80 $\mathrm{m}$ on average) and crossed by the depression of the Lama. The clay soil remains soggy and muddy in the rainy season. Water stagnates for several months in this region. The hydrographic network comprises 3 large rivers which are the Mono in the east, the Zio and the Haho in the center with several small tributaries that flow into the "lac Togo" (Fig. 1). All these streams have a low flow, closely linked to seasonal variations of precipitations [33].

\section{Laboratory confirmation}

Sample collection: Samples were collected according to standardized procedures as previously described $[9,10]$. Briefly, fine needle aspirates (FNA) were collected from the center of non-ulcerative lesions or from undermined edges of ulcerative lesions including necrotic tissue. Swabs were collected by circling the entire undermined edges of ulcerative lesions. Samples taken were put in tubes containing cell lysis solution (CLS, Qiagen, Hilden, Germany) and sent for PCR analysis at INH.
Laboratory testing: Direct smears for microscopy were prepared from swab and FNA samples at peripheral care units or CNRT-UB and subjected to Ziehl-Neelsen staining for detection of acid fast bacilli at the laboratory of the CHR. Slides were analyzed by microscopy according to the WHO [34] recommended grading system. All slides were double checked at the INH by a second technician for external quality control.

All molecular analyses were conducted at the NRL for BU at INH as previously described [10]. For PCR analysis, DNA was extracted from FNA and swab samples with the Gentra Puregene DNA extraction kit (Qiagen) with minor modifications of the manufacturer's protocol. The conventional IS2404-PCR with gel-based amplicon detection was applied using dry-reagent-based consumables (DRB-IS2404 PCR). Briefly, for the DRB-PCR, the primers MU5 (AGCGACCCCAGTGGATTGGT) and MU6 (CGGTGATCAAGCGTTCACGA) were lyophilized in reaction tubes. The Illustra PuReTaq Ready-ToGo PCR beads (GE Healthcare) containing Taq 
polymerase, dNTPs and $\mathrm{Mg}^{2+}$ were added and dissolved in water before adding DNA template. All PCR assays included negative extraction, positive, negative (no template) and inhibition controls. All inhibited samples were repeated after 10 -fold dilution.

\section{Sample size}

We used the power calculation tool of Epi-Info (version $7 ; 2012$ ) to determine the sample size by setting $\alpha$ to $5 \%$ and power $\beta$ to $80 \%$. The health and population development survey (EDST; 2014) in Togo showed that $36,2 \%$ of households use water from unprotected sources [35, 36]. The odds ratio (OR) of the association between cases and controls was set at 2, yielding a sample size of $100 \mathrm{BU}$ cases and 200 controls.

\section{Data collection}

The survey was conducted by a team of four people including a clinician from CHR Tsévié, a focal point of the national program of BU surveillance, a community volunteer and a laboratory technician. Case residence was identified by the community volunteer. Once at home, we selected one or two matched control subjects. A well-structured questionnaire was administered to all selected participants (Additional file 1: Questionnaire form S1). For participants who could not respond in French, the interview was conducted in the local language. For children under 10 years, we interviewed their parents mainly for their activities and behavior. All the participants gave their consent prior to data collection on socio-demographic characteristics, behavior, occupational and environmental factors as well as administration of BCG vaccination.

\section{Statistical analysis}

Data collected were entered in a database designed through Epi-Info software (Version 7; 2012). Statistical analysis was carried out by SPSS software (Statistical Package for Social Science, Version 16.0, SPSS Inc. and Chicago, IL). Qualitative data were presented as number $\mathrm{n}$ (\%) and quantitative data as mean \pm standard deviation. Buruli ulcer was considered as the dependent variable and socio-demographic characteristics, occupational and environmental factors as independent variables. Student $\mathrm{t}$-test was used for comparison of mean or median age and number of people in the household between patients and controls with significant level set at $p \leq 0.05$. Univariate logistic regression was used to determine the risk factors of $M$. ulcerans infection by determining the odds ratio (OR) and 95\% confidence interval (CI). All variables obtained from the univariate analysis with $p$ value $\leq 0.1$ were retained for the multivariate model. The final model was obtained after a step-by-step backward elimination step using multiple logistic regressions.

\section{Results}

Clinical diagnosis, laboratory confirmation and characteristics of BU cases.

During the study period, 129 probable cases were observed (Table 1). ZN microscopy confirmed the presence of acid-fast bacilli (AFB) in 67 (52\%) among probable cases while PCR detected $M$. ulcerans DNA in 91 cases (71\%). The two techniques were both positive in 58 cases (44.5\%) and no AFB were detected from any of the PCR negative lesions (Table 1). Of all confirmed cases, lesions were mainly ulcers (41.7\%), nodules $(27.5 \%)$ and plaques (19.8\%) (Table 2). Most of these lesions were found on the lower (40\%) and the upper limbs (45\%). The rest of lesions were localized on the buttocks, abdomen, back and head. Of 91confirmed cases, 83 (91\%) responded to the questionnaire. The remaining 8 cases were absent at the time of the survey. Therefore, the case-control study was carried out with 83 cases and 128 control subjects. The socio-demographic characteristics of the participants are presented in Table 3.

\section{Univariate analysis \\ Socio-demographic characteristics of the participants}

Most of BU patients (66\%) were under 15 years of age and were significantly younger (median age $=11$ years) compared to controls (median age $=19$ years with $73 \%$ of who aged more than 15 years $)(p=0.001)$ (Table 3). The primary school educational level was more frequent $(59 \%) \quad(p=0.03)$ in children aged $\leq 10$ years $(28.2 \%)$ while the secondary school educational level was associated with the 15-24 age groups $(56.2 \%) \quad(p=0.007) \quad$ (Table 3). Among cases, women $(60 \%)$ were more frequently affected than men $(40 \%)$ $(p=0.01)$. There was no significant difference in the number of people living per household between cases and controls $(p=0.58)$ (Table 3).

Table 1 Yearly distribution of clinically suspected BU cases, laboratory tests used for confirmation and positive BU cases detected in Zio and Yoto Districts of Maritime Region, Togo, March 2013 to April 2015

\begin{tabular}{ll}
\hline Parameters & $\begin{array}{l}\text { Number of } \\
\text { BU suspected cases, n }\end{array}$ \\
\hline Yearly distribution of BU suspected cases & \\
2013 (March to December) & 31 \\
2014 & 63 \\
2015 (January to April) & 35 \\
Total & 129 \\
Laboratory confirmation tests & Positive BU Cases, n (\%) \\
Ziehl-Neelsen microscopy & $67(51.9)$ \\
(129 cases analyzed) & \\
PCR technique (129 cases analyzed) & 91 (70.5) \\
ZN microscopy and IS2404 PCR & 58 (44.4) \\
\hline
\end{tabular}


Table 2 Type and localization of observed lesions in 91 BU cases in Zio and Yoto Districts of Maritime Region, Togo, March 2013-May 2015

\begin{tabular}{ll}
\hline Clinical Characteristics & Number of BU cases, $\mathrm{n}(\%)$ \\
\hline Type of lesions & $10(10.9)$ \\
Edema & $25(27.5)$ \\
Nodule & $18(19.8)$ \\
Plaque & $38(41.7)$ \\
Ulcer & $\mathbf{9 1 ( 1 0 0 . 0 )}$ \\
Total & \\
Localization of lesions & $3(3.0)$ \\
Abdomen & $2(2.5)$ \\
Back & $3(3.0)$ \\
Buttocks & $2(2.5)$ \\
Head & $37(40.6)$ \\
Lower limbs & $44(48.3)$ \\
Upper limbs & $\mathbf{9 1 ( 1 0 0 . 0 )}$ \\
Total &
\end{tabular}

\section{Environmental factors}

\section{Exposure to water contact}

We found that drinking or washing clothes with water taken from rivers $(p=0.95)$, open boreholes $(p=0.98)$ and boreholes with pump $(p=0.49)$ were not associated with an increased risk of contracting Buruli ulcer (Table 4). However, bathing with water from an open borehole was associated with higher risk of contracting $\mathrm{BU}(\mathrm{OR}=5.07$, 95\% CI $=1.33-19.31$ ) (Table 4). The frequent use of soap while bathing was not associated with reduced risk of BU $(p=0.69)$. In contrary, a significant decrease of risk of $M$. ulcerans infection was observed when using detergents for washing clothes or dishes $(\mathrm{OR}=0.38,95 \% \mathrm{CI}=0.32-0.45)$ (Table 4). Walking in stagnant water or wading in mud did not significantly increase risk of $M$. ulcerans infection $(p=0.72)$. However, frequently crossing a river $(\mathrm{OR}=1.93$, $95 \% \mathrm{CI}=1.09-3.39)$ or swimming $(\mathrm{OR}=1.98,95 \%$ $\mathrm{CI}=1.11-3.52)$ in a river were associated with an increased risk of BU. Receiving cuts or scratches $(\mathrm{OR}=1.88,95 \%=1.06-3.36)$ near rivers represented an additional increasing risk for contracting BU (Table 4).

\section{Exposure to insects}

Our study showed that receiving insect bites near a river was significantly increase risk of $M$. ulcerans infection $(\mathrm{OR}=2.13,95 \% \mathrm{CI}=1.19-3.83)$ (Table 4$)$. This risk was higher when it occurred on the forearm $(\mathrm{OR}=1.88,95 \%$ $\mathrm{CI}=1.08-3.31)$, the arm $(\mathrm{OR}=1.77,95 \% \mathrm{CI}=1.01-3.10)$ and the hands $(\mathrm{OR}=1.79,95 \% \mathrm{CI}=1.03-3.14)$ compared to the other parts of the body. We found that mosquito bites at home were not associated with an
Table 3 Socio-demographic characteristics of the participants of the case-control study in Zio and Yoto Districts of the Maritime Region, Togo, May 19-30, 2015

\begin{tabular}{|c|c|c|c|c|}
\hline Characteristics & $\begin{array}{l}\text { Cases n } \\
(\%)\end{array}$ & $\begin{array}{l}\text { Controls n } \\
(\%)\end{array}$ & $\begin{array}{l}\text { Total n } \\
(\%)\end{array}$ & $p^{*}$ \\
\hline Number of participants & $83(39.3)$ & $128(60.7)$ & 211 & \\
\hline Sex & & & & 0.32 \\
\hline Female & $50(60.2)$ & $68(53.1)$ & $118(55.9)$ & \\
\hline Male & 33 (39.8) & $60(46.9)$ & $93(44.1)$ & \\
\hline \multicolumn{5}{|l|}{ Age } \\
\hline $\begin{array}{l}\text { Median } \\
\text { (range in years) }\end{array}$ & $11(3-65)$ & $19(8-60)$ & $15(3-65)$ & 0.001 \\
\hline$<10$ & $39(47.0)$ & $15(11.7)$ & $54(25.6)$ & 0.01 \\
\hline $11-14$ & $16(19.3)$ & 19 (14.8) & $35(16.6)$ & 0.03 \\
\hline $15-24$ & $13(15.7)$ & $47(36.7)$ & $60(28.4)$ & 0.76 \\
\hline$>=25$ & $15(18.1)$ & $47(36.7)$ & $62(29.4)$ & \\
\hline District of residence & & & & 0.75 \\
\hline Zio & $62(74.7)$ & $98(76.6)$ & $160(75.8)$ & \\
\hline Yoto & $21(25.3)$ & $30(23.4)$ & $51(24.2)$ & \\
\hline Education level & & & & 0.03 \\
\hline None & $19(22.9)$ & $36(28.1)$ & $55(26.1)$ & \\
\hline Primary school & $57(68.7)$ & $67(52.3)$ & $124(58.8)$ & \\
\hline Secondary school & $7(8.4)$ & $25(19.5)$ & $32(15.2)$ & \\
\hline Ethnicity & & & & 0.48 \\
\hline Ewe & $76(96.2)$ & $110(97.3)$ & $186(96.9)$ & \\
\hline $\begin{array}{l}\text { Other (Lamba. } \\
\text { Moba et Peulh) }\end{array}$ & $3(3.8)$ & $3(2.7)$ & $6(3.1)$ & \\
\hline
\end{tabular}

Number of people in household

Median (range)

$8.5(2-40) \quad 8.0(3-40)$

$8.0(2-40)$

*Boldface type indicates differences that were statistically significant at $p<0.05$ between cases and controls

increased risk of $M$. ulcerans infection $(p=0.90)$ (Table 4). The use of mosquito coils $(p=0.27)$ or bednets $(p=0.26)$ did not provide any significant reduction in the risk of contracting BU (Table 4).

\section{Farming activities}

Farming (93.8\%) was the main activity of the participants of the study. However, there was no significant difference in practicing this activity between patients and controls $(p=0.78)$ (Table 4). In addition, some tasks such as sowing $(\mathrm{OR}=0.29,95 \% \mathrm{CI}=0.09-0.90)$ or harvesting $(\mathrm{OR}=0.19,95 \% \mathrm{CI}=0.05-0.75)$ during farming showed significant decrease in the risk of contracting $\mathrm{BU}$ (Table 4). Frequently wearing trousers $(\mathrm{OR}=0.36,95 \%$ $\mathrm{CI}=0.20-0.65)$ or a hat $(\mathrm{OR}=0.21,95 \% \mathrm{CI}=0.08-0.52)$ while performing farming activities provided significant reduction in the risk. However, wearing clothes at home or in non-farming activities did not provided any significant reduction in the risk of BU disease (Table 4). 
Table 4 Univariate analysis of risk factors for Buruli ulcer disease in Zio and Yoto districts of Maritime Region, Togo, May 19-30, 2015

\begin{tabular}{|c|c|c|c|c|}
\hline Characteristics & Cases n (\%) & Controls n (\%) & Univariable OR (95\% Cl) & $p^{*}$ \\
\hline Farming & $77(92.8)$ & $120(93.8)$ & $0.86(0.29-2.56)$ & 0.78 \\
\hline \multicolumn{5}{|c|}{ Farming activities } \\
\hline Plowing & 79 (83.1) & $117(91.4)$ & $0.46(0.19-1.01)$ & 0.07 \\
\hline Sowing & $73(88.0)$ & $123(96.1)$ & $0.29(0.09-0.90)$ & 0.03 \\
\hline Harvesting & $74(89.2)$ & $125(97.7)$ & $0.19(0.05-0.75)$ & 0.01 \\
\hline
\end{tabular}

Exposure to water

Primary source of drinking water

$38(45.8)$

58 (45.3)

$1.02(0.58-1.77)$

0.95

Open borehole

$15(18.1)$

$23(18.0)$

$1.0(0.49-2.01)$

0.98

Borehole with pump

75 (90.4)

$119(93.0)$

$0.71(0.26-1.92)$

0.49

Primary source of washing water

River or stream

$42(50.6)$

$60(46.9)$

$1.16(0.68-2.02)$

0.59

Open borehole

28 (21.9)

$1.21(0.63-2.31)$

0.56

Borehole with pump

$116(90.6)$

$0.75(0.31-1.83)$

0.54

Bathing with a water from an open borehole

Standing water in house

$3(2.3)$

$5.07(1.33-19.31)$

0.01

Swam, waded or bathed in a river or stream

$18(14.1)$

$0.86(0.38-1.97)$

0.72

Crossed a body of water

37 (28.9)

$1.98(1.11-3.52)$

0.02

Received cuts, scratches and thorn pricks near a river

55 (43.0)

$1.93(1.09-3.39)$

0.02

$49(62.8)$

$60(47.2)$

$1.88(1.06-3.36)$

0.03

Exposure to insect bite

Received insect bite near a river

$50(64.9)$

59 (46.5)

$2.13(1.19-3.83)$

0.01

location of insect bite on the body

Head

48 (57.8)

56 (43.8)

$1.76(1.01-3.08)$

0.05

Forearms

57 (44.5)

$1.88(1.08-3.31)$

0.03

Arms

$1.77(1.01-3.10)$

0.04

Hands

57 (44.5)

$1.79(1.03-3.14)$

0.04

trunk

57 (44.5)

$1.70(0.98-2.98)$

0.06

thigh

56 (43.8)

$1.76(1.01-3.08)$

0.05

Legs

57 (44.5)

$1.70(0.98-2.98)$

0.06

Feet

57 (44.5)

$1.70(0.98-2.98)$

0.06

Mosquito bite in house

$124(96.9)$

$0.95(0.48-1.89)$

0.90

Exposure to animals

Owned livestock or pets

$60(77.9)$

$100(78.7)$

$0.95(0.48-1.89)$

0.89

Handled livestock or pets

26 (20.6)

$0.71(0.33-1.50)$

0.37

Share indoor living space with livestock or pets

$37(29.4)$

$1.15(0.62-2.14)$

0.65

Bitten or scratched by animals

$10(7.8)$

$0.84(0.27-2.56)$

Exposure to infectious agents

BCG vaccination

$41(51.2)$

$64(50.4)$

$1.03(0.59-1.181)$

0.90

Soap use while bathing

Sometimes

$5(6.4)$

$10(7.9)$

Always

73 (93.6)

$117(92.1)$

$0.80(0.26-2.43)$

0.69

Soap use while washing

Sometimes

$5(6.0)$

$0(0.0)$

Always

78 (94.0)

$128(100.0)$

$0.38(0.32-0.45)$

0.01 
Table 4 Univariate analysis of risk factors for Buruli ulcer disease in Zio and Yoto districts of Maritime Region, Togo, May 19-30, 2015 (Continued)

\begin{tabular}{|c|c|c|c|c|}
\hline Characteristics & Cases n (\%) & Controls n (\%) & Univariable OR (95\% Cl) & $p^{*}$ \\
\hline \multicolumn{5}{|l|}{ Clothing worn while farming } \\
\hline Trousers & $40(48.2)$ & $92(71.9)$ & $0.36(0.20-0.65)$ & 0.001 \\
\hline Top shirt & $76(91.6)$ & $120(93.8)$ & $0.72(0.25-2.01)$ & 0.55 \\
\hline Closed shoes & $9(10.8)$ & $26(20.3)$ & $0.48(0.21-1.08)$ & 0.07 \\
\hline Dress & $34(41.0)$ & $48(37.5)$ & $1.16(0.66-2.03)$ & 0.61 \\
\hline Open shoes & $73(88.0)$ & $110(85.9)$ & $1.19(0.522-2.73)$ & 0.67 \\
\hline Hat & $6(7.2)$ & $35(27.3)$ & $0.21(0.08-0.52)$ & 0.001 \\
\hline \multicolumn{5}{|l|}{ Clothing worn in non-farming activity } \\
\hline Trousers & $33(39.8)$ & $61(47.7)$ & $0.72(0.41-1.27)$ & 0.26 \\
\hline Top shirt & $73(88.0)$ & $118(92.2)$ & $0.62(0.25-1.56)$ & 0.31 \\
\hline Closed shoes & $4(4.8)$ & $5(3.9)$ & $1.25(0.32-4.78)$ & 0.74 \\
\hline Dress & $33(39.8)$ & $54(42.2)$ & $0.90(0.51-1.59)$ & 0.73 \\
\hline Open shoes & $72(86.7)$ & $114(89.1)$ & $0.80(0.35-1.87)$ & 0.61 \\
\hline Hat & $1(1.2)$ & $7(5.5)$ & $0.21(0.25-1.75)$ & 0.15 \\
\hline \multicolumn{5}{|l|}{ Insect protection products use } \\
\hline Sometimes & $74(96.1)$ & $118(92.2)$ & 1 & 0.27 \\
\hline Always & $3(3.9)$ & $10(7.8)$ & $0.59(0.22-1.64)$ & \\
\hline \multicolumn{5}{|l|}{ Bednets use } \\
\hline Sometimes & $40(51.3)$ & $76(59.4)$ & 1 & \\
\hline Always & $38(48.7)$ & $52(40.6)$ & $0.72(0.41-1.27)$ & 0.26 \\
\hline Perception and etiology of the BUD & $68(88.3)$ & $113(88.3)$ & $1.00(0.42-2.42)$ & 0.99 \\
\hline \multicolumn{5}{|l|}{ Behavior and beliefs } \\
\hline Poor hygiene cause Buruli ulcer & $57(81.4)$ & $116(91.3)$ & $0.42(0.17-0.99)$ & 0.04 \\
\hline Seeking treatment with plants & $4(5.3)$ & $10(7.9)$ & $0.64(0.19-2.12)$ & 0.47 \\
\hline
\end{tabular}

*Boldface type indicates differences that were statistically significant at $p<0.05$ between cases and controls

\section{Exposure to animals}

In our study, we found that living with $(p=0.89)$ or sharing indoor living space with livestock $(p=0.37)$ did not represent a significant increase in the risk of $M$. ulcerans infection neither did incurring bites or scratches from $(p=0.76)$ (Table 4$)$. Also, hunting or handling of wild animals $(p=0.65)$ was not significantly associated with an increasing risk of BU infection.

\section{BCG vaccination}

Most of participants showed BCG vaccine scars and there was no significant difference between cases and controls $(p=0.90)$ (Table 4$)$.

\section{Attitude, behavior and beliefs of BUD}

Of the participants interviewed, $88.3 \%$ were familiar with $\mathrm{BU}$ symptoms and this attitude was similar between BU cases and controls $(p=0.99)$. Regarding treatment behaviors, most of cases (83.5\%) indicated seeking help from hospital while $5.3 \%$ believed in herbal treatment as the first preferred treatment option (Table 4). Considering the hygiene practice, BU cases as well as controls thought that personal poor hygiene and dirty surroundings could increase the risk of contracting BU (Table 4).

\section{Multivariate analysis}

After adjustment for potential confounders, we found that factors such as age $(<10$ years $(\mathrm{aOR}=11.48,95 \% \mathrm{CI}=$ $3.72-35.43)$ and 10 to 14 years $(\mathrm{aOR}=3.63,95 \% \mathrm{CI}=$ $1.22-10.83)$ ), receiving insect bites near a river in children aged 10 to14 years $(\mathrm{aOR}=7.8,95 \% \mathrm{CI}=(1.48-41.24))$ and bathing with water from open borehole $(\mathrm{aOR}=5.77,95 \%$ $\mathrm{CI}=1.11-29.27$ ) (Table 5) remain as potential factors of increasing risk of $M$. ulcerans infection.

\section{Discussion}

The objective of this study was to identify risk factors for Buruli ulcer in the two endemic districts of Zio and Yoto of the Maritime region. This is the first study that has investigated these factors in Togo. In general, socio-demographic, behavioral or environmental factors have 
Table 5 Multivariate model for risk factors of Buruli ulcer disease in Zio and Yoto Districts of the Maritime Region, Togo, May 19-30, 2015

\begin{tabular}{|c|c|c|}
\hline Characteristics & $\mathrm{aOR}(95 \% \mathrm{Cl})$ & $p^{*}$ \\
\hline \multicolumn{3}{|l|}{ Age (Years) } \\
\hline$<10$ & $11.48(3.72-35.43)$ & 0.001 \\
\hline $11-14$ & $3.63(1.22-10.83)$ & 0.02 \\
\hline $15-24$ & $1.07(0.39-2.97)$ & 0.88 \\
\hline$>25$ & 1 & \\
\hline \multicolumn{3}{|l|}{ Receiving insect bites near a river (Yes/No) } \\
\hline$<10$ (years) & $3.29(0.77-14.04)$ & 0.11 \\
\hline $11-14$ (Years) & $7.80(1.48-41.21)$ & 0.016 \\
\hline 15-24 (Years) & $3.05(0.71-12.99)$ & 0.13 \\
\hline$>25$ (Years) & $1.76(0.48-6.45)$ & 0.39 \\
\hline Bathing with water from open borehole & $5.77(1.11-29.27)$ & 0.03 \\
\hline
\end{tabular}

*Boldface type indicates differences that were statistically significant at $p<$ 0.05 between cases and controls

been considered as important risk factors for $M$. ulcerans infection.

\section{Socio-demographic factors}

The present study showed that children under 15 years of age were at higher risk of contracting Buruli ulcer than adults. This result is in accordance with other studies conducted in Benin [11] and Ivory Coast [13] as well as WHO reports [37]. Indeed, in this age group children appeared to be often less protected especially at the head and feet [11]. Also, children's behavior is usually driven by their parents' activities as they accompanied them to the river for washing and for farming where they were highly exposed to aquatic areas that are associated with an increasing risk of BU infection.

\section{Environmental factors}

We found that bathing with water from an open borehole was associated with higher risk of contracting BU. Similar results were found in Ghana [38], Ivory Coast [39] and Cameroon [14]. Indeed, other studies [6, 28, 40] have also shown that using unprotected water sources for bathing was associated with M. ulcerans infection. It has also been observed that even when used with soap, unprotected water sources constitute an increased risk of M. ulcerans infection [37]. However, Raghunathan et al. [38] in Ghana found that using a detergent while bathing provides significant reduction in Buruli ulcer risk. This difference could be explained by the antibacterial power of the soap used. Besides, in our study people from villages commonly used the local soap. On the other hand, we found that using soap to wash clothes or dishes was reducing the risk. This time, the type of the soap used for the laundry is provided from commercial brands which are strongly enriched in detergents and acids. Our study also identified other water sources of $M$. ulcerans infection such as swimming in a river, frequently crossing a river, receiving insect bites or injuries of cuts near rivers. However, after adjustment for potential confounders, only receiving insect bites near a river remained as an independent predictor of acquiring BU infection. Similar results were found in Ghana [38] but in Ivory Coast [13] and in other study [12], it was found that swimming or wading in water did significantly increase the risk of $\mathrm{BU}$ infection. To explore the difference of our finding with other studies, we looked to determine any potential age confounding or effect modification. Therefore, we found that insect bites increase the risk of $\mathrm{BU}$ only in 101-14 years age group $(\mathrm{aOR}=7.80, \quad 95 \% \mathrm{CI}=$ 1.48-41.21). Though, other studies did not determine in which age group swimming or wading in water significantly increased the risk of BU, we could explain the difference between these studies by the age of BU cases. Further, in our study, $63 \%$ of BU cases were aged $<15$ years while in Ivory Coast, $75 \%$ of cases were aged more than 15 years who are able to swim or wad in a river.

Most of the people surveyed were perform agricultural activities. However, we did not find any significant association with the risk of contracting BU. Among agricultural activities, planting and harvesting activities were associated with decrease risk of M. ulcerans infection. Similar results were found in Cameroon [14]. We observed that wearing a long-sleeved shirt or a long dress while performing agricultural activities did not provide significant reduction of the risk of contracting of Buruli ulcer. This observation is in accordance with the study conducted in Cameroon [14]. On the other hand, we found that wearing pants or hats is associated with reduction in the risk of mycobacterial infection. This would explain the low frequency of wounds on head and legs observed in our investigation. These results are consistent with those found in Ghana [12, 38] and Ivory Coast [13].

In Australia, Lavender et al. [20] showed that mosquito bites were significantly associated with Buruli ulcer. However, we did not find any risk of M. ulcerans infection associated to mosquito bites in Togo. In general, results of studies on mosquito bites associated with the use of mosquito coils or bednets during $M$. ulcerans infection are often contradictory $[11,14,38,39,41]$.

Some studies [12, 13, 42] have shown that animals such as chickens, goats, cats and pigs could harbour $M$. ulcerans and exposure to these animals may increase the risk of contracting BU disease. During this study, we did not observe significant increase in risk of contracting BU associated with contact with domestic animals.

BCG vaccine is delivered against a mycobacterium. This vaccination could therefore provide a cross- 
protection against $M$. ulcerans infection [43]. In our study, we did not observe any significant difference in the percentage of BCG vaccination scar between patients and controls. The lack of a significant association with BCG vaccination with $M$. ulcerans infection has been also described in the literature [12, 16, 43]. However, data from Benin [11], Ivory Coast [13] and Cameroon [14] showed negative correlation between BCG vaccination and BU. Studies conducted to explore this possible cross-protection have often led to contradictory results. Indeed, a multicenter study [44] conducted in the DR Congo, Ghana and Togo did not reveal any significant association between BCG vaccination and BU disease.

\section{Attitude, behavior and belief on BU}

The attitude of the participants interviewed has considerably improved with their capacity to recognize some BU symptoms and their ability to refer suspected cases to medical treatment compared to the situation 5 years before [33]. This finding could be attributable to several awareness campaigns in the community that had influenced their behavior toward this disease [33]. However, there remains some effort to help recognizing early symptoms by the community as well as the herbalists because $5.3 \%$ of $\mathrm{BU}$ patients continue to believe in herbal treatment as the first preferred treatment option. Poor individual hygiene and dirty surrounding were recognized as a potential risk factor for participants in the present study. The impact of poor hygiene and its possible role as a risk factor has been underlined in studies in Benin [11, 45] and Ghana [12].

This study had some limitation. We did not reach all participants especially some BU cases due to their unavailability during the survey time. The sample size was calculated based on the proportion of households using water from unprotected sources which was higher than the prevalence of $\mathrm{BU}$. The number of newly confirmed BU cases in Togo every year is low and varies from 30 to 65 patients. During the study period, we found $91 \mathrm{BU}$ cases but 8 patients were not available at the survey time. The main concern with the limit number of controls was due to the fact that in many households, there were often two to three patients and exceptionally in one house up to six. In those households, it was difficult to enroll two folds of controls. Moreover, as $47 \%$ of BU patients were under 10 years, it was difficult to interview children who were not capable to describe their activities which are driven by their parent's duties. The reason we had decided to use their parents as controls sometimes.

\section{Conclusions}

Our study identified some significant risk factors for BU infection including age, bathing with water from open boreholes and receiving insect bites near a river in Zio and Yoto Districts of the Maritime Region in south Togo.

\section{Additional file}

Additional file 1: Questionnaire form S1. Questionnaire form used to collect data during the survey on risk factor for Mycobacterium ulcerans infection in Zio and Yoto districts of Maritime Region, Togo, May 19-30, 2017. (DOC $84 \mathrm{~kb}$ )

\section{Abbreviations}

ASC: Health community volunter; BCG: Bacille calmette et guérin; CHR: Centre hospitalier régional; CNRT-UB: Centre national de référence pour le traitement de I'ulcère de buruli; DAHW-Togo: Association allemande de lutte contre la lèpre; DITM: Department of infectious and tropical medicine; EDST: Enquête de développement sanitaire du Togo; ESTBA: Ecole supérieure des techniques biologiques et alimentaires; INH: Institut national d'hygiène; OR: Odds Ratio; PCR: Polymerase chain reaction; PNLUB-LP: Programme national de lutte contre I'ulcère de buruli, la lèpre et le pian; SPSS: Statistical package for social science; VIH: Human immunodeficiency virus; WHO: World Health Organisation

\section{Acknowledgements}

We would like sincerely to thank the Institut National d'Hygiène through its Director for supporting financially this study and ensuring the logistic of the investigation. We also express our acknowledgments to the National Program for the Control of Buruli Ulcer, Leprosy and Pian through its Coordinator for authorizing the survey. Many thanks to the CNTR-UB and PNLU-LP focal points.

Funding

Not applicable

\section{Availability of data and materials}

All data generated or analyzed during this study are included in this published article [and its supplementary information files]. However, the datasets used and/or analyzed during the current study are available from the corresponding author on reasonable request.

\section{Authors' contributions}

$\mathrm{IM}, \Pi \mathrm{T}$ and DSK contributed to the study design, statistical analyses of data and wrote the.

paper. EP, KK were involved in the field investigation, data collection and reviewing the manuscript. $\mathrm{MB}$ provides advice for study methodology and performed critical review of the manuscript for important scientific content. $\mathrm{KA}, \mathrm{GB}$ and FXW contributed to the critical review of the document. YK, KB and $\mathrm{ABK}$ contributed to the facilitation of the project, participated in its design, coordination and review the paper. All authors read and approved the final manuscript.

\section{Ethics approval and consent to participate}

The study protocol was approved by the National Program for Buruli Ulcer Control, (Authorization No.006/2014/MS/DGS/DSSP/PNLUB-LP) and the Ministry of Health as an integral part of the surveillance of the disease. However, this study did not require a review of the ethics committee. The objectives of the study were explained to the participants and their inclusion was voluntary. For each participant, we obtained a signed consent. As for children, parents or legal representatives gave consent on their behalf.

\section{Consent for publication}

Not applicable

Competing interests

All authors declare that they have no competing interests.

\section{Publisher's Note}

Springer Nature remains neutral with regard to jurisdictional claims in published maps and institutional affiliations. 


\section{Author details}

'Institut National d'Hygiène (INH), National Reference Laboratory for Buruli ulcer disease in Togo, 26 QAD Rue Nangbeto, 1BP, 1396 Lomé, Togo. ${ }^{2}$ Ecole Supérieure des Techniques Biologiques et Alimentaires (ESTBA), Laboratoire des Sciences Biologiques et des Substances Bioactives, Université de Lomé, Lomé, Togo. ${ }^{3}$ Centre National de Référence pour le Traitement de l'Ulcère de Buruli (CNRT-UB), Centre Hospitalier Régional (CHR) de Tsévié, Lomé, Togo. ${ }^{4}$ Department for Infectious Diseases and Tropical Medicine (DITM), Medical Center of the University of Munich (LMU), Munich, Germany. ${ }^{5}$ Programme National de Lutte Contre I'Ulcère de Buruli, la Lèpre et le Pian (PNLUB-LP), Lomé, Togo. ${ }^{6}$ German Leprosy and Tuberculosis Relief Association (DAHW-T), Togo office, Lomé, Togo

Received: 14 August 2017 Accepted: 11 January 2018

Published online: 19 January 2018

\section{References}

1. WHO | Buruli ulcer. http://www.who.int/mediacentre/factsheets/fs199/en/. Accessed 16 Oct 2016

2. Junghanss T, Johnson RC, Pluschke G. In: Hotez PJ FJ, editor. Mycobacterium ulcerans disease. 23rd ed: Mansons Trop Dis; 2014. p. 519-31.

3. WHO: Treatment of mycobacterium ulcerans disease (Buruli ulcer): guidance for health workers. 2012. http://apps.who.int/iris/handle/10665/77771. Accessed 23 Apr 2017

4. Darie H. Mycobacterium ulcerans infection: epidemiological, clinical and therapeutical aspects. Bull Société Pathol Exot 1990. 2003;96(5):368-71.

5. Huang GKL, Johnson PD. Epidemiology and management of Buruli ulcer. Expert Rev Anti-Infect Ther. 2014;12(7):855-65.

6. Hotez PJ, Kamath A. Neglected tropical diseases in sub-Saharan Africa: review of their prevalence, distribution, and disease burden. Cappello M, editor. PLoS Negl Trop Dis. 2009;3(8):e412

7. Meyers WM, Tignokpa N, Priuli GB, Portaels F. Mycobacterium ulcerans infection (Buruli ulcer): first reported patients in Togo. Br J Dermatol. 1996; 134(6):1116-21.

8. Portaels F, Chemlal K, Elsen P, Johnson PD, Hayman JA, Hibble J, et al. Mycobacterium ulcerans in wild animals. Rev Sci Tech Int Off Epizoot. 2001; 20(1):252-64.

9. Bretzel G, Huber KL, Kobara B, Beissner M, Piten E, Herbinger K-H, et al. Laboratory confirmation of Buruli ulcer disease in Togo, 2007-2010. PLoS Negl Trop Dis. 2011;5(7):e1228.

10. Beissner M, Huber KL, Badziklou K, Halatoko WA, Maman I, Vogel F, et al. Implementation of a national reference laboratory for Buruli ulcer disease in Togo. PLoS Negl Trop Dis. 2013;7(1):e2011.

11. Debacker M, Portaels F, Aguiar J, Steunou C, Zinsou C, Meyers W, et al. Risk factors for Buruli ulcer, Benin. 2006. https://www.ncbi.nlm.nih.gov/pubmed/ 17073079. Accessed 15 Jan 2018.

12. Kenu E, Nyarko KM, Seefeld L, Ganu V, Käser M, Lartey M, et al. Risk factors for Buruli ulcer in Ghana-a case control study in the Suhum-KraboaCoaltar and Akuapem south districts of the eastern region. Phillips $\mathrm{RO}$ editor. PLoS Negl Trop Dis. 2014:8(11):e3279.

13. N'krumah RTAS, Koné B, Tiembre I, Cissé G, Pluschke G, Tanner M, et al. Socio-environmental factors associated with the risk of contracting Buruli ulcer in Tiassalé, south Côte d'Ivoire: a case-control study. Johnson C, editor. PLoS Negl Trop Dis. 2016:10(1):e0004327.

14. Pouillot R, Matias G, Wondje CM, Portaels F, Valin N, Ngos F, et al. Risk factors for Buruli ulcer: a case control study in Cameroon. Small P, editor. PLoS Negl Trop Dis. 2007;1(3):e101.

15. Quek TYJ, Athan E, Henry MJ, Pasco JA, Redden-Hoare J, Hughes A, et al. Risk factors for mycobacterium ulcerans infection, southeastern Australia. Emerg Infect Dis. 2007;13(11):1661-6.

16. Bratschi MW, Ruf M-T, Andreoli A, Minyem JC, Kerber S, Wantong FG, Pritchard J, et al. "Mycobacterium Ulcerans persistence at a village water source of Buruli ulcer patients." edited by Christian Johnson. PLoS Negl Trop Dis. 2014;8(3):e2756. doi: https://doi.org/10.1371/journal.pntd.0002756.

17. Tian, Diangoné RB, Niamké $S$, Tissot-Dupont $H$, Drancourt M. Detection of Mycobacterium Ulcerans DNA in the environment, Ivory Coast. PLoS One. 2016;11(3):e0151567.

18. Williamson HR, Benbow ME, Campbell LP, Johnson CR, Sopoh G, Barogui Y, Merritt RW, Small PLC. "Detection of mycobacterium Ulcerans in the environment predicts prevalence of Buruli ulcer in Benin." edited by Joseph
M. Vinetz. PLoS Negl Trop Dis. 2012;6(1):e1506. doi: https://doi.org/10.1371/ journal.pntd.0001506.

19. Williamson HR, Benbow ME, Nguyen KD, Beachboard DC, Kimbirauskas RK McIntosh MD, Quaye C, et al. "Distribution of mycobacterium Ulcerans in Buruli ulcer endemic and non-endemic aquatic sites in Ghana." edited by Mathieu Picardeau. PLoS Negl Trop Dis. 2008;2(3):e205. doi: https://doi.org/ 10.1371/journal.pntd.0000205.

20. Lavender CJ, Fyfe JAM, Azuolas J, Brown K, Evans RN, Ray LR, et al. Risk of Buruli ulcer and detection of mycobacterium ulcerans in mosquitoes in southeastern Australia. PLoS Negl Trop Dis. 2011;5(9):e1305.

21. Stinear T, Davies JK, Jenkin GA, Hayman JA, Oppedisano F, Johnson PD. Identification of mycobacterium ulcerans in the environment from regions in Southeast Australia in which it is endemic with sequence capture-PCR. Appl Environ Microbiol. 2000;66(8):3206-13.

22. Marsollier L, Aubry J, Milon G, Brodin P. Aquatic insects and transmission of mycobacterium ulcerans. Med Sci MS. 2007;23(6-7):572-5.

23. Eddyani M, Ofori-Adjei D, Teugels G, De Weirdt D, Boakye D, Meyers WM, et al. Potential role for fish in transmission of mycobacterium ulcerans disease (Buruli ulcer): an environmental study. Appl Environ Microbiol. 2004;70(9):5679-81.

24. Marsollier L, Stinear T, Aubry J, Saint André JP, Robert R, Legras P, et al. Aquatic plants stimulate the growth of and biofilm formation by mycobacterium ulcerans in axenic culture and harbor these bacteria in the environment. Appl Environ Microbiol. 2004;70(2):1097-103.

25. Durnez $L$, Suykerbuyk $P$, Nicolas $V$, Barrière $P$, Verheyen $E$, Johnson $C R$, et al. Terrestrial small mammals as reservoirs of mycobacterium ulcerans in benin Appl Environ Microbiol. 2010;76(13):4574-7.

26. Portaels F, Meyers WM, Ablordey A, Castro AG, Chemlal K, et al. First cultivation and characterization of mycobacterium ulcerans from the environment. PLoS Negl Trop Dis. 2008;2:e178.

27. Jacobsen $\mathrm{KH}$, Padgett JJ. Risk factors for mycobacterium ulcerans infection. Int J Infect Dis. 2010;14(8):e677-81.

28. Merritt RW, Walker ED, Small PLC, Wallace JR, Johnson PDR, Benbow ME, et al. Ecology and transmission of Buruli ulcer disease: a systematic review. Phillips RO, editor. PLoS Negl Trop Dis. 2010;4(12):e911.

29. Duker AA, Portaels F, Hale M. Pathways of Mycobacterium ulcerans infection: A review. Environ Int. 2006:32(4):567-73.

30. Beissner M, Piten E, Maman I, Symank D, Jansson M, Nitschke J, et al. Spontaneous clearance of a secondary Buruli ulcer lesion emerging ten months after completion of chemotherapy - a case report from Togo. Phillips RO, editor. PLoS Negl Trop Dis. 2012;6(7):e1747.

31. Jansson M, Beissner M, Phillips RO, Badziklou K, Piten E, Maman I, et al. Comparison of two assays for molecular determination of Rifampin resistance in clinical samples from patients with Buruli ulcer disease. J Clin Microbiol. 2014;52(4):1246-9.

32. Beissner M, Arens N, Wiedemann F, Piten E, Kobara B, Bauer M, et al. Treatment outcome of patients with Buruli ulcer disease in Togo. PLoS Negl Trop Dis. 2015;9(10):e0004170. doi: https://doi.org/10.1371/journal.pntd. 0004170.

33. Rapport Analyse situation UB Togo.doc: http://proadiph.org/IMG/pdf/ analyse_de_situation_de_la_lutte_contre_l_ulcere_de_burili_dans_la_ region_maritime_au_togo.pdf. Accessed 23 Oct 2016.

34. WHO. Laboratory diagnosis of Buruli ulcer. Manual for health personnel2014: http://apps.who.int/iris/bitstream/10665/111738/1/9789241505703_ eng.pdf. Accessed 15 Jan 2018.

35. Principaux Indicateurs de Santu00E9 2013 Final1x - pb-annuelle-pissanitaire-tg-2013.pdf. http://www.stat-togo.org/contenu/pdf/pb/pbannuelle-pis-sanitaire-tg-2013.pdf. Accessed 23 Oct 2016.

36. Togo Enquête Démographique et de Santé 2013-2014 [FR301] - FR301.pdf. https://dhsprogram.com/pubs/pdf/FR301/FR301.pdf. Accessed 23 Oct 2016.

37. OMS | Ulcère de Buruli. http://www.who.int/mediacentre/factsheets/fs199/ $\mathrm{fr} /$. Accessed 16 Oct 2016.

38. Raghunathan PL, Whitney EAS, Asamoa K, Stienstra Y, Taylor TH, Amofah GK et al. Risk factors for Buruli ulcer disease (mycobacterium ulcerans infection): results from a case-control study in Ghana. Clin Infect Dis Off Publ Infect Dis Soc Am. 2005;40(10):1445-53.

39. Ahoua L, Guetta AN, Ekaza E, Bouzid S, N'Guessan R, Dosso M. Risk factors for Buruli ulcer in Côte d'Ivoire: results of a case-control study, august 2001. Afr J Biotechnol. 2009;8(4). https://www.ajol.info/index.php/ajb/article/view/ 59860/48134. Accessed 15 Jan 2018.

40. Walsh DS, Portaels F, Meyers WM. Buruli ulcer (Mycobacterium ulcerans infection). Trans R Soc Trop Med Hyg. 2008;102(10):969-78. 
41. Landier J, Boisier P, Fotso Piam F, Noumen-Djeunga B, Simé J, Wantong FG, et al. Adequate wound care and use of bed nets as protective factors against Buruli ulcer: results from a case control study in Cameroon. Zhou XN, editor. PLoS Negl Trop Dis. 2011;5(11):e1392.

42. Walsh DS, Portaels F, Meyers WM. Buruli ulcer: advances in understanding mycobacterium ulcerans infection. Dermatol Clin. 2011;29(1):1-8.

43. Zogo B, Djenontin A, Carolan K, Babonneau J, Guegan J-F, Eyangoh S, et al. A field study in Benin to investigate the role of mosquitoes and other flying insects in the ecology of mycobacterium ulcerans. PLoS Negl Trop Dis. 2015;9(7):e0003941.

44. Phillips RO, Phanzu DM, Beissner M, Badziklou K, Luzolo EK, Sarfo FS, et al. Effectiveness of routine BCG vaccination on Buruli ulcer disease: a casecontrol study in the Democratic Republic of Congo, Ghana and Togo. PLoS Negl Trop Dis 2015; 9(1). http://www.ncbi.nlm.nih.gov/pmc/articles/ PMC4287572/. Accessed 23 Oct 2016.

45. Johnson RC, Boni G, Barogui Y, Sopoh GE, Houndonougbo M, Anagonou E, et al. Assessment of water, sanitation, and hygiene practices and associated factors in a Buruli ulcer endemic district in Benin (West Africa). BMC Public Health. 2015; 15(1). Available from: http://www.biomedcentral.com/14712458/15/801. Accessed 13 Oct 2016

Submit your next manuscript to BioMed Central and we will help you at every step:

- We accept pre-submission inquiries

- Our selector tool helps you to find the most relevant journal

- We provide round the clock customer support

- Convenient online submission

- Thorough peer review

- Inclusion in PubMed and all major indexing services

- Maximum visibility for your research

Submit your manuscript at www.biomedcentral.com/submit
Biomed Central 